\title{
POLYNOMIAL AUTOMORPHIC FORMS AND NONDISCONTINUOUS GROUPS
}

\author{
BY \\ MARVIN ISADORE KNOPP(1)
}

1. Introduction. Let $\mathscr{H}$ denote the upper half-plane and let $\Gamma$ be a group of linear fractional transformations preserving $\mathscr{H}$. Then every element $M$ of $\Gamma$ can be represented in the form

$$
M \tau=(a \tau+b) /(c \tau+d) ; a, b, c, d \text { real, } a d-b c=1 .
$$

With the linear fractional transformation we associate the two matrices

$$
M= \pm\left(\begin{array}{ll}
a & b \\
c & d
\end{array}\right)
$$

and we note that composition of transformations corresponds to matrix multiplication. As a matter of convenience, we will interchangeably think of $M$ as a linear fractional transformation and as a matrix.

A function $f(\tau)$, meromorphic in $\mathscr{H}$, such that

$$
f(M \tau)=f(\tau), \text { for all } M \in \Gamma, \tau \in \mathscr{H}
$$

is said to be an automorphic function with respect to $\Gamma$. (The usual definition of automorphic function imposes a growth condition on $f(\tau)$ as $\tau$ approaches certain points of the real axis from within an angle. The same remark is applicable in connection with the definition of automorphic form, given below. It will be apparent from the nature of the results given here that this growth condition plays no role in the present context.) Let $r$ be a real number. A function $f(\tau)$, meromorphic in $\mathscr{H}$, is said to be an automorphic form of dimension $r$ on $\Gamma$, with multiplier system $v$, provided

$$
f(M \tau)=v(M)(c \tau+d)^{-r} f(\tau)
$$

for every

$$
M=\left(\begin{array}{ll}
* & * \\
c & d
\end{array}\right) \in \Gamma, \quad \tau \in \mathscr{H}
$$

Received by the editors June 6, 1965 .

(1) Research supported in part by a National Science Foundation grant at the University of Wisconsin. 
where $v(M)$ depends only on $M$ and not on $\tau$, and $|v(M)|=1$ for every $M \in \Gamma$. Here a branch of $(c \tau+d)^{-r}$ is fixed once and for all. We see that an automorphic function on $\Gamma$ is an automorphic form of dimension 0 on $\Gamma$, with multiplier system $v \equiv 1$.

Suppose, as before, that $\Gamma$ is a group of linear fractional transformations preserving $\mathscr{H}$. We say that $\alpha$ is a limit point of $\Gamma$ provided that there is a point $z$ and a sequence $\left\{V_{n}\right\}$ of distinct elements of $\Gamma$ such that $V_{n} z \rightarrow \alpha$, as $n \rightarrow \infty$. If $\alpha$ is not a limit point it is called an ordinary point of $\Gamma$. $\Gamma$ is said to be discontinuous at $\alpha$ if $\alpha$ is an ordinary point of $\Gamma$. Note that the $V_{n} z$ need not be distinct. Thus, in particular, if $\alpha$ is a fixed point of infinitely many distinct $V \in \Gamma$, then $\alpha$ is a limit point of $\Gamma$.

$\Gamma$ is discontinuous in $\mathscr{H}$ if every point of $\mathscr{H}$ is an ordinary point of $\Gamma$. It has been known since Poincaré that $\Gamma$ is discontinuous in $\mathscr{H}$ if and only if $\Gamma$ is discrete in the topology defined by elementwise convergence (cf. [3, p. 99]). In particular then in order to show that $\Gamma$ is discontinuous in $\mathscr{H}$ it suffices to show that there does not exist an infinite sequence of distinct $V_{n}$ converging to $I$, the identity matrix. In view of this remark, there is

THEOREM A. Suppose $\Gamma$ is a group of linear fractional transformations preserving $\mathscr{H}$, and suppose there is a nonconstant function $f(z)$ automorphic with respect to $\Gamma$. Then $\Gamma$ is discontinuous in $\mathscr{H}$.

Proof. Suppose $V_{n} \rightarrow I$, with $V_{n}$ distinct elements of $\Gamma$. Consider the countable set $W_{i j}$ of elements of $\Gamma$ given by $W_{i j}=V_{i}^{-1} V_{j}$, for $i \neq j, 1 \leqq i, j<\infty$. Then no $W_{i j}$ is equal to $I$. Hence there exists $z_{0} \in H$ such that $z_{0}$ is fixed by no $W_{i j}$, since every linear fractional transformation $\neq I$ has at most two distinct fixed points. It follows that the points $V_{n} z_{0}$ are all distinct, for it $V_{i} z_{0}=V_{j} z_{0}$, then $V_{i}^{-1} V_{j} z_{0}=z_{0}$. On the other hand $V_{n} z_{0} \rightarrow z_{0}$ since $V_{n} \rightarrow I$. But $f\left(V_{n} z_{0}\right)=f\left(z_{0}\right)$ for all $n$, and since $f$ is meromorphic at $z_{0}, f(z)=f\left(z_{0}\right)$ for all $z \in \mathscr{H}$.

Since $f(z)$ is not constant in $\mathscr{H}$ it follows that $\Gamma$ is discrete and therefore discontinuous in $\mathscr{H}$.

J. R. Smart has raised the question of whether we can replace $f(z)$ by an automorphic form of dimension $r$ on $\Gamma$ and still conclude that $\Gamma$ is discontinuous. The answer as we shall see is "no." However, we find that the nondiscontinuous groups $\Gamma$ that can occur are limited in variety and we shall classify all of them (Theorem 13). It is clear that the above simple proof of Theorem A fails if $f(z)$ is an automorphic form instead of an automorphic function. Nevertheless there is the following result which is a natural generalization of Theorem A for automorphic forms of integral dimension with multiplier system $v \equiv 1$ on $\Gamma$.

THEOREM 1. Suppose $\Gamma$ is a group of linear fractional transformations preserving $\mathscr{H}$. Then $\Gamma$ is discontinuous if one of the following holds. 
(a) There is an automorphic form $f(\tau)$ of positive even integral dimension on $\Gamma$, with multiplier system $v \equiv 1$, such that $f(\tau)$ is not a polynomial.

(b) There is an automorphic form $f(\tau)$ of positive odd integral dimension on $\Gamma$, with multiplier system $v \equiv \pm 1$, such that $f(\tau)^{2}$ is not polynomial.

Condition (a) is equivalent to the existence of an automorphic form $g(\tau)$ of negative even integral dimension on $\Gamma$, with multiplier system $v \equiv 1$, such that $g(\tau)$ is not the reciprocal of a polynomial. For $f(\tau)$ is an automorphic form of dimension $r$ on $\Gamma$ if and only if $1 / f(\tau)$ is an automorphic form of dimension $-r$ on $\Gamma$. For the same reason, condition (b) is equivalent to the existence of an automorphic form $g(\tau)$ of negative odd integral dimension on $\Gamma$, with multiplier system $v \equiv \pm 1$, such that $g^{2}(\tau)$ is not the reciprocal of a polynomial.

We give a proof of Theorem 1 in $\S 2$. As we shall see the condition that $f(\tau)$ is not a polynomial $\left(f(\tau)^{2}\right.$ is not a polynomial in (b)) is not superfluous. That is, there do exist groups $\Gamma$ which are not discontinuous and which support automorphic forms of the type excluded in (a) and (b). We find all groups $\Gamma$ which support polynomial automorphic forms, and in the process all groups $\Gamma$ which are not discontinuous, but support automorphic forms of integral dimension with multiplier system $v \equiv 1$ (Theorems 7 and 8). Next, we find all nondiscontinuous groups $\Gamma$ which support automorphic forms of integral dimension with multiplier system $v$ not necessarily $\equiv 1$, and at the same time we describe all such automorphic forms on these groups (Theorem 10). Finally we describe all nondiscontinuous groups $\Gamma$ which support automorphic forms of real dimension (Theorem 13).

It is not too difficult to see that all of our methods and results go through if we drop the condition $|v(M)| \equiv 1$ on the multiplier systems and also allow $r$ to be complex. We do not carry out the details here, however.

Walter Roelcke, by different methods has obtained results which overlap with those given here. His work is unpublished.

2. Proof of Theorem 1. In the proof of Theorem 1 we need

LEMMA 2. Let $r$ be a nonnegative integer and let $F(\tau)$ be a complex function. Let $a, b, c, d$ be complex numbers with $a d-b c=1$. Then

$$
\frac{d^{r+1}}{d \tau^{r+1}}\left\{(c \tau+d)^{r} F\left(\frac{a \tau+b}{c \tau+d}\right)\right\}=(c \tau+d)^{-r-2} \frac{d^{r+1}}{d(M \tau)^{r+1}} F\left(\frac{a \tau+b}{c \tau+d}\right), M \tau=\frac{a \tau+b}{c \tau+d},
$$

for any $\tau$ such that both sides exist.

RemarK. This result was first discovered by G. Bol [1]. If $F$ is assumed to be regular in a neighborhood of $(a \tau+b) /(c \tau+d)$, the lemma is a simple consequence of Cauchy's integral formula. Without the assumption of regularity, a proof can be given by induction on $r$. We give a proof via Cauchy's formula.

Proof. By the Cauchy formula, the left hand side of the statement of the lemma is equal to 


$$
\frac{1}{2 \pi i} \int_{C} \frac{(c \zeta+d)^{r} F\left(\frac{a \zeta+b}{c \zeta+d}\right)}{(\zeta-\tau)^{r+2}} d \zeta
$$

where $C$ is a simple closed curve with $\tau$ in its interior. We make the change variable $\zeta^{\prime}=(a \zeta+b) /(c \zeta+d)$ and the above integral becomes

$$
\begin{aligned}
\frac{1}{2 \pi i} \int_{C^{\prime}} \frac{F\left(\zeta^{\prime}\right)}{\left(-c \zeta^{\prime}+a\right)^{r+2}\left(\frac{d \zeta^{\prime}-b}{-c \zeta^{\prime}+a}-\tau\right)^{r+2}} d \zeta^{\prime} \\
\quad=\frac{1}{2 \pi i} \int_{C^{\prime}} \frac{F\left(\zeta^{\prime}\right)}{\left.\left\{\zeta^{\prime}(c \tau+d)-(a \tau+b)\right\}\right\}^{r+2}} d \zeta^{\prime} \\
\quad=(c \tau+d)^{-r-2} \frac{1}{2 \pi i} \int_{C^{\prime}} \frac{F\left(\zeta^{\prime}\right)}{\left(\zeta^{\prime}-\frac{a \tau+b}{c \tau+d}\right)^{r+2}} d \zeta^{\prime},
\end{aligned}
$$

where $C^{\prime}=M(C)$ is a simple closed curve with $(a \tau+b) /(c \tau+d)$ in its interior. By the Cauchy formula again, this last integral is equal to

$$
(c \tau+d)^{-r-2} \frac{d^{r+1}}{d(M \tau)^{r+1}} F\left(\frac{a \tau+b}{c \tau+d}\right)=(c \tau+d)^{-r-2} F^{(r)}\left(\frac{a \tau+b}{c \tau+d}\right) .
$$

COROLlARY 3. Suppose that $\Gamma$ is a group of real linear fractional transformations with determinant one, and $r$ is a nonnegative integer. If $f(\tau)$ is an automorphic form on $\Gamma$ of dimension $r$, with multiplier system $v$, then $f^{(r+1)}(\tau)=\left(d^{r+1} / d \tau^{r+1}\right)(f(\tau))$ is an automorphic form on $\Gamma$ of dimension $-r-2$, with multiplier system $v$.

Proof. For every

we have

$$
M=\left(\begin{array}{ll}
* & * \\
c & d
\end{array}\right) \in \Gamma
$$

$$
(c \tau+d)^{r} f(M \tau)=v(M) f(\tau) .
$$

Differentiating both sides $r+1$ times with respect to $\tau$ and using Lemma 2, we obtain.

$$
(c \tau+d)^{-r-2} f^{(r+1)}(M \tau)=v(M) f^{(r+1)}(\tau),
$$

the desired conclusion.

Proof of Theorem 1. From now on we assume that $\Gamma$ is normalized so that it consists of real linear fractional transformations of determinant one. We consider case (a) first. By Corollary $3, f^{(r+1)}(\tau)$ is an automorphic form of dimension $-r-2$ 
with $v \equiv 1$. A simple calculation shows that $(f(\tau))^{r+2}\left(f^{(r+1)}(\tau)\right)^{r}$ is an automorphic function on $\Gamma$. Suppose $\Gamma$ is not discontinuous on $\mathscr{H}$. We will show that then $f(\tau)$ is a polynomial.

By Theorem A, $(f(\tau))^{r+2}\left(f^{(r+1)}(\tau)\right)^{r}=K_{1}$, a constant. If $f(\tau)$ is not a polynomial, $K_{1} \neq 0$, so that $f(\tau)$ (and also $f^{(r+1)}(\tau)$ ) has no zero in $\mathscr{H}$. Hence $g(\tau)=(f(\tau))^{1 / \boldsymbol{r}}$ is a well-defined meromorphic function in $\mathscr{H}$ if we choose a branch. Furthermore since

we have

$$
(c \tau+d)^{r} f(M \tau)=f(\tau), \text { for every } M=\left(\begin{array}{ll}
* & * \\
c & d
\end{array}\right) \in \Gamma,
$$

$$
(c \tau+d) g(M \tau)=v_{0}(M) g(\tau), v_{0}^{r} \equiv 1
$$

and therefore $g^{3}(\tau)$ is an automorphic form of dimension 3, with multiplier system $v_{0}^{3}$. Also by Corollary $3, g^{\prime \prime}(\tau)$ is an automorphic form of dimension -3 , with multiplier system $v_{0}$. Hence $g^{\prime \prime}(\tau) g^{3}(\tau)$ is an automorphic form of dimension zero and multiplier system $v_{0}^{4}$, so that, finally, $\left(g^{\prime \prime}(\tau) g^{3}(\tau)\right)^{r}$ is an automorphic function. Hence by Theorem A, $\left(g^{\prime \prime}(\tau) g^{3}(\tau)\right)^{r}=K_{2}^{*}$, a constant. But if $K_{2}^{*}=0$, then $g^{\prime \prime}(\tau) \equiv 0$; we conclude that $g(\tau)$, and therefore also $f(\tau)$, is a polynomial. Hence $K_{2}^{*} \neq 0$, and we write $g^{\prime \prime}(\tau) g^{3}(\tau)=K_{2} \neq 0$, with a fixed branch chosen.

Let $h(\tau)=g^{2}(\tau)$. Then $h(\tau)$ is an automorphic form of dimension 2 and multiplier system $v_{0}^{2} ; h^{\prime \prime \prime}(\tau)$ is a form of dimension -4 and multiplier system $v_{0} ; h^{2}(\tau) h^{\prime \prime \prime}(\tau)$ is a form of dimension 0 and multiplier system $v_{0}^{5}$. Furthermore, then $\left(h^{2}(\tau) h^{\prime \prime \prime}(\tau)\right)^{r}$ is an automorphic function, so that by Theorem $\mathrm{A}\left(h^{2}(\tau) h^{\prime \prime \prime}(\tau)\right)^{r}=K_{3}^{*}$, or $h^{2}(\tau) h^{\prime \prime \prime}(\tau)=K_{3}$, with a particular branch chosen. We show that $K_{3} \neq 0$. If $K_{3}=0$, then $h^{\prime \prime \prime}(\tau) \equiv 0$, so that $h(\tau)$ is a polynomial. Hence $f(\tau)=\left(g^{2}(\tau)\right)^{r / 2}=h(\tau)^{r / 2}$ is a polynomial, contrary to assumption. Thus $K_{3} \neq 0$.

Since $g^{\prime \prime}(\tau)=K_{2} g^{-3}(\tau)$, we have $g^{\prime \prime \prime}(\tau)=-3 K_{2} g^{-4}(\tau) g^{\prime}(\tau)$. On the other hand $h(\tau)=g(\tau)^{2}$ implies that $h^{\prime \prime \prime}(\tau)=2 g(\tau) g^{\prime \prime \prime}(\tau)+6 g^{\prime}(\tau) g^{\prime \prime}(\tau)$. Therefore,

$$
\frac{1}{2} h(\tau)^{2} h^{\prime \prime \prime}(\tau)=g(\tau)^{4}\left\{g(\tau) g^{\prime \prime \prime}(\tau)+3 g^{\prime}(\tau) g^{\prime \prime}(\tau)\right\}=\frac{1}{2} K_{3}
$$

Hence,

$$
\begin{aligned}
g(\tau) g^{\prime \prime \prime}(\tau) & =\frac{1}{2} K_{3} g^{-4}(\tau)-3 g^{\prime}(\tau) g^{\prime \prime}(\tau) \\
& =\frac{1}{2} K_{3} g^{-4}(\tau)-3 g^{\prime}(\tau) \cdot K_{2} g^{-3}(\tau),
\end{aligned}
$$

or

$$
g^{\prime \prime \prime}(\tau)=\frac{1}{2} K_{3} g^{-5}(\tau)-3 K_{2} g^{-4}(\tau) g^{\prime}(\tau)
$$

On the other hand, since $g^{\prime \prime}(\tau)=K_{2} g^{-3}(\tau)$, we have $g^{\prime \prime \prime}(\tau)=-3 K_{2} g^{-4}(\tau) g^{\prime}(\tau)$. Thus we conclude that $K_{3}=0$, a contradiction. Hence $\Gamma$ is discontinuous. 
Case (b) now follows from case (a) if we replace $f(\tau)$ by $f(\tau)^{2}$.

3. Remarks on linear fractional transformations. We here summarize some relevant facts concerning linear fractional transformations. The proofs of these facts can be found in [3, pp. 69-70].

A linear fractional transformation

$$
M=\left(\begin{array}{ll}
a & b \\
c & d
\end{array}\right) ; a, b, c, d \text { real, } a d-b c=1, M \neq I,
$$

is called elliptic, hyperbolic or parabolic according as $|a+d|<2,|a+d|>2$, or $|a+d|=2$. If $M$ is parabolic then $M$ has exactly one fixed point $\tau_{0}, \tau_{0}$ is real, and $M$ has the form

$$
\begin{aligned}
\frac{1}{\tau^{\prime}-\tau_{0}} & =\frac{1}{\tau-\tau_{0}}+c, \quad c \text { real, if } \tau_{0} \neq \infty, \\
\tau^{\prime} & =\tau+c, \quad c \text { real, if } \tau_{0}=\infty
\end{aligned}
$$

Here $\tau^{\prime}=M \tau$. If $M$ is elliptic or hyperbolic, $M$ has two distinct fixed points $\tau_{1}, \tau_{2}$. If $M$ is elliptic $\tau_{2}=\bar{\tau}_{1} \neq \tau_{1}$; if $M$ is hyperbolic, $\tau_{1}$ and $\tau_{2}$ are real. In either case $M$ has the form

$$
\frac{\tau^{\prime}-\tau_{1}}{\tau^{\prime}-\tau_{2}}=\kappa \frac{\tau-\tau_{1}}{\tau-\tau_{2}}, \quad \tau^{\prime}=M \tau
$$

Here $\kappa$ is a complex number; furthermore, writing $\kappa=\rho e^{i \theta}, \rho>0,0 \leqq 0<2 \pi$, we have

(i) $M$ is elliptic if and only if $\rho=1, \theta \neq 0$ and

(ii) $M$ is hyperbolic if and only if $\rho \neq 1, \theta=0$.

It is worth noting that $M^{n}=I$ if and only if $\kappa^{n}=1$. Thus if $M$ is periodic, it must be elliptic, but not conversely.

If $\Gamma$ consists entirely of hyperbolics (elliptics) fixing $\tau_{1}$ and $\tau_{2}$, then $\Gamma$ is discontinuous if an only if the set of $\kappa$ 's corresponding to the elements of $\Gamma$ has no limit point. This is immediate from the definition of discontinuity of a group.

4. Polynomial automorphic forms. Theorem 1 shows that, in an attempt to find all nondiscontinuous groups that support automorphic forms of integral dimension with multiplier system $v \equiv \pm 1$, we can restrict our attention to groups which support polynomial automorphic forms of positive even integral dimension with multiplier system $v \equiv 1$. In this section we investigate polynomial automorphic forms and the groups which support them. Throughout this section $r$ will be a positive integer and $p(\tau) \neq 0$ a polynomial automorphic form of dimension $r$ with multiplier system $v \equiv 1$ on $\Gamma$.

LEMma 4. If $\Gamma \neq\{I\}$, then the degree of $p(\tau)$ is less than or equal to $r$. 
Proof. Suppose $p(\tau)$ has degree $r^{*}>r$, with leading coefficient $a_{r^{*}} \neq 0$. Let

$$
M=\left(\begin{array}{ll}
a & b \\
c & d
\end{array}\right) \in \Gamma .
$$

If $c \neq 0$, then $(c \tau+d)^{r} p(M \tau)$ is not a polynomial and therefore $\neq p(\tau)$. Hence $c=0$, and

$$
M=\left(\begin{array}{ll}
a & b \\
0 & d
\end{array}\right), a d=1,
$$

or

$$
M=\left(\begin{array}{ll}
d^{-1} & b \\
0 & d
\end{array}\right) .
$$

Then $M \tau=\tau / d^{2}+b / d$. Now $M \in \Gamma$ implies $d^{r} p\left(\tau / d^{2}+b / d\right)=p(\tau)$. The leading coefficient in $d^{r} p\left(\tau / d^{2}+b / d\right)$ is $d^{r-2 r^{*}} \cdot a_{r^{*}}$. Hence $d^{r-2 r^{*}}=1, d= \pm 1$. Therefore $M= \pm I$, as a matrix. Hence $M=I$, as a linear fractional transformation.

THEOREM 5. Suppose $p(\tau)$ has at least three distinct zeros. Then $\Gamma$ consists solely of elliptic elements of order at most $r+1$.

Proof. Since $p(\tau)$ is entire, we conclude that

$$
(c \tau+d)^{r} \cdot p(M \tau)=p(\tau), \text { for } M=\left(\begin{array}{ll}
* & * \\
c & d
\end{array}\right) \in \Gamma,
$$

holds in the entire plane, by analytic continuation. Writing

$$
M^{v}=\left(\begin{array}{ll}
a_{v} & b_{v} \\
c_{v} & d_{v}
\end{array}\right)
$$

$v$ a positive integer, we observe that $M^{v}\left(\tau_{0}\right)=\infty$ if and only if $c_{v} \tau_{0}+d_{v}=0$.

Suppose $M \in \Gamma$. Then $M$ has at most two fixed points; we can choose $\tau_{0}$ such that $p\left(\tau_{0}\right)=0$ and $M \tau_{0} \neq \tau_{0}$. Consider the sequence $\tau_{0}, M \tau_{0}, \cdots, M^{r+1} \tau_{0}$. If these are all distinct, then at most one of these is the point at $\infty$. But

$$
0=p\left(\tau_{0}\right)=\left(c_{v} \tau_{0}+d_{v}\right)^{r} p\left(M^{v} \tau_{0}\right), \quad 0 \leqq v \leqq r+1 .
$$

Thus, with at most one exceptional value of $v, p\left(M^{v} \tau_{0}\right)=0$, and $p(\tau)$ has at least $r+1$ zeros, a contradiction to Lemma 4. It follows that there exist $i$ and $j$, $0 \leqq i<j \leqq r+1$, such that $M^{j} \tau_{0}=M^{i} \tau_{0}$, or $M^{j-i} \tau_{0}=\tau_{0}$. Thus $M^{j-i}$ has as least three distinct fixed points if $M$ is elliptic or hyperbolic, and at least two if $M$ is parabolic. In either case $M^{j-i}=I$. Hence $M$ has order at most $r+1$ and is therefore elliptic.

COROLlaRY 6. Under the conditions of Theorem $5, \Gamma$ is finite and a fortiori discontinuous. 
Proof. By a special case of a theorem of Burnside [2, p. 251] every periodic subgroup of $\operatorname{GL}(2, C)$, of bounded period is finite. Here $C$ is the complex field. Under the conditions of Lemma $5, \Gamma$ is periodic of period $(r+1)$ !. Now $\Gamma$ can be thought of as a periodic subgroup of $\operatorname{GL}(2, C)$, of period $(2 r+2)$ !. Hence $\Gamma$ is finite.

We next consider the case when $p(\tau)$ has exactly two zeros.

THEOREM 7. Let $p(\tau)$ have degree $r_{0} \leqq r$ and suppose $p(\tau)$ has exactly two distinct zeros $\tau_{1}$ and $\tau_{2}$. Write $p(\tau)=A\left(\tau-\tau_{1}\right)^{\alpha}\left(\tau-\tau_{2}\right)^{r_{0}-\alpha}$ where $\alpha$ is an integer such that $0<\alpha<r_{0}$.

(a) If $\tau_{1}$ and $\tau_{2}$ are real and $r=r_{0}=2 \alpha, \Gamma$ contains at most hyperbolics fixing $\tau_{1}$ and $\tau_{2}$ and elliptics of order two interchanging $\tau_{1}$ and $\tau_{2}$.

(b) If $\tau_{2}=\bar{\tau}_{1}$ and $r=r_{0}=2 \alpha, \Gamma$ consists at most of elliptic elements, possibly of infinite order, fixing $\tau_{1}$ and $\tau_{2}$.

(c) If $\tau_{1}$ and $\tau_{2}$ (or $\bar{\tau}_{1}$ and $\bar{\tau}_{2}$ ) are both in $\mathscr{H}$, and $r+r_{0}=2 \alpha, \Gamma$ contains at most elliptic elements of order two interchanging $\tau_{1}$ and $\tau_{2}$.

(d) If $\tau_{1}$ and $\tau_{2}$ are real and $r=2 r_{0}-\alpha, r_{0}=2 \alpha$, then $\Gamma$ consists of at most elliptic elements of order three permuting the points $\tau_{1}, \tau_{2}$, and $\infty$.

(e) If $\tau_{1}$ is real, $\tau_{2}$ is not real, and $r-r_{0}=\alpha, \Gamma$ contains at most elliptic elements of order two fixing $\tau_{2}$ and interchanging $\tau_{1}$ and $\infty$.

(f) If $r_{0}=r \neq 2 \alpha$, and $\tau_{2}=\bar{\tau}_{1}, \Gamma$ consists of at most elliptic elements of order $r-2 \alpha\left(r / 2-\alpha\right.$, if $r$ is even) fixing $\tau_{1}$ and $\tau_{2}$.

(g) Otherwise $\Gamma=\{I\}$.

In cases (c) and (f). $\Gamma$ is finite, In case (d), $\Gamma$ is a cyclic group of order three. In case (e), $\Gamma$ is a cyclic group of order two. In these cases $\Gamma$ is discontinuous. In cases (a) and (b), $\Gamma$ need not be discontinuous.

Proof. Without loss of generality we may assume $A=1$. The argument used to prove Theorem 5 shows that $\Gamma$ has no parabolic elements, since a parabolic linear fractional transformation is not of finite order and has only one fixed point.

Suppose $M \in \Gamma$, with $M$ hyperbolic. Then, once again by the argument of Theorem 5, $M \tau_{1}=\tau_{1}$, and $M \tau_{2}=\tau_{2}$, since $M$ is not a finite order. It follows that $\tau_{1}$ and $\tau_{2}$ are real and $M$ has the form

$$
\tau^{\prime}=M \tau, \quad \frac{\tau^{\prime}-\tau_{1}}{\tau^{\prime}-\tau_{2}}=\kappa \frac{\tau-\tau_{1}}{\tau-\tau_{2}},
$$

with $\kappa$ real, $\kappa>0, \kappa \neq 1$. A short calculation yields $M \tau=(a \tau+b) /(c \tau+d)$, $a d-b c=1$, with

$$
\begin{array}{ll}
a=\frac{\tau_{1}-\kappa \tau_{2}}{\kappa^{1 / 2}\left(\tau_{1}-\tau_{2}\right)}, & b=\frac{\tau_{1} \tau_{2}(\kappa-1)}{\kappa^{1 / 2}\left(\tau_{1}-\tau_{2}\right)}, \\
c=\frac{(1-\kappa)}{\kappa^{1 / 2}\left(\tau_{1}-\tau_{2}\right)}, & d=\frac{\kappa \tau_{1}-\tau_{2}}{\kappa^{1 / 2}\left(\tau_{1}-\tau_{2}\right)} .
\end{array}
$$


By the transformation formula for $p(\tau)$,

$$
\begin{aligned}
\left(\tau-\tau_{1}\right)^{\alpha}\left(\tau-\tau_{2}\right)^{r_{0}-\alpha} & =p(\tau)=(c \tau+d)^{r} p(M \tau) \\
& =(c \tau+d)^{r}\left(M \tau-\tau_{1}\right)^{\alpha}\left(M \tau-\tau_{2}\right)^{r_{0}-\alpha} \\
& =(c \tau+d)^{r-r_{0}}\left(a-c \tau_{1}\right)^{\alpha}\left(a-c \tau_{2}\right)^{r_{0}-\alpha}\left(\tau-\tau_{1}\right)^{\alpha}\left(\tau-\tau_{2}\right)^{r_{0}-\alpha} .
\end{aligned}
$$

Hence $r=r_{0}$ and $\left(a-c \tau_{1}\right)^{\alpha}\left(a-c \tau_{2}\right)^{r_{0}-\alpha}=1$. By (1) this latter condition becomes $\kappa^{r / 2-\alpha}=1$. Since $M$ is hyperbolic $r=2 \alpha$; otherwise $\kappa$ would be a root of unity, a contradiction.

Suppose $M \in \Gamma$, with $M$ elliptic. If $M$ is not of finite order, we conclude that $\tau_{1}$ and $\tau_{2}$ are the fixed points of $M$, and therefore $\tau_{2}=\bar{\tau}_{1} \neq \tau_{1}$. Then the argument of the last paragraph shows that $r=r_{0}=2 \alpha$. If $M$ is elliptic of finite order, this argument yields no information.

Suppose

$$
M=\left(\begin{array}{ll}
a & b \\
c & d
\end{array}\right) \in \Gamma,
$$

where $M \neq I$ is elliptic of finite order. If $c=0$, then $a d=1$ so that $|a+d| \geqq 2$, contrary to the fact that $M$ is elliptic. Hence $c \neq 0$ and the transformation formula for $p(\tau)$ can be written

$$
\begin{aligned}
(c \tau+ & d)^{r}\left(\frac{a \tau+b}{c \tau+d}-\tau_{1}\right)^{\alpha}\left(\frac{a \tau+b}{c \tau+d}-\tau_{2}\right)^{r_{0}-\alpha} \\
& =(c \tau+d)^{r-r_{0}}\left\{\tau\left(a-c \tau_{1}\right)+b-d \tau_{1}\right\}^{\alpha} \cdot\left\{\tau\left(a-c \tau_{2}\right)+b-d \tau_{2}\right\}^{r_{0}-\alpha} \\
& =c^{r-r_{0}}(\tau+d / c)^{r-r_{0}}\left\{\tau\left(a-c \tau_{1}\right)+b-d \tau_{1}\right\}^{\alpha} \cdot\left\{\tau\left(a-c \tau_{2}\right)+b-d \tau_{2}\right\}^{r_{0}-\alpha} \\
& =\left(\tau-\tau_{1}\right)^{\alpha}\left(\tau-\tau_{2}\right)^{r_{0}-\alpha}
\end{aligned}
$$

Suppose first that $a-c \tau_{1}=0$, that is, that $M^{-1} \tau_{1}=\infty$. Then of course $\tau_{1}=a / c$ and $a-c \tau_{2} \neq 0$. Then, by (2), $\left(b-d \tau_{1}\right)^{\alpha}\left(a-c \tau_{2}\right)^{r-\alpha} c^{r-r 0}=1$, and either

$$
r-r_{0}=\alpha, \quad \tau_{1}=-d / c, \quad M^{-1} \tau_{2}=\tau_{2},
$$

or

$$
r_{0}-\alpha=\alpha, \quad r-r_{0}=r_{0}-\alpha, \quad M^{-1} \tau_{2}=\tau_{1}, \quad \tau_{2}=-d / c .
$$

If (3) holds, we have $M \tau_{1}=\infty, M \tau_{2}=\tau_{2}$, and $M^{-1} \tau_{1}=\infty$. Hence $M^{2} \tau_{1}=\tau_{1}$, so that $M^{2}=1$. Also $\tau_{2}$ is not real and $r \neq r_{0}$.

If (4) holds, $r_{0}=2 \alpha$ and $r=3 \alpha$. Also, $M \tau_{1}=\tau_{2}, M \tau_{2}=\infty$, and $M(\infty)=\tau_{1}$. Hence $M^{3} \tau_{1}=M^{2} \tau_{2}=M(\infty)=\tau_{1}$, and we conclude that $M^{3}=I$. In this case $\tau_{1}$ and $\tau_{2}$ are real.

Clearly the same reasoning applies if $a-c \tau_{2}=0$, that is, if $M^{-1} \tau_{2}=\infty$. Hence we may assume next that $a-c \tau_{1} \neq 0, a-c \tau_{2} \neq 0$. In this case (2) becomes 


$$
\begin{gathered}
c^{r-r_{0}}\left(a-c \tau_{1}\right)^{\alpha}\left(a-c \tau_{2}\right)^{r_{0}-\alpha}(\tau+d / c)^{r-r_{0}}\left(\tau-M^{-1} \tau_{1}\right)^{\alpha}\left(\tau-M^{-1} \tau_{2}\right)^{r_{0}-\alpha} \\
=\left(\tau-\tau_{1}\right)^{\alpha}\left(\tau-\tau_{2}\right)^{r_{0}-\alpha .}
\end{gathered}
$$

Then $r=r_{0},\left(a-c \tau_{1}\right)^{\alpha}\left(a-c \tau_{2}\right)^{r_{0}-\alpha}=1$, and either

$$
M^{-1} \tau_{1}=\tau_{2}, \quad M^{-1} \tau_{2}=\tau_{1}, \quad \alpha=r-\alpha
$$

or

$$
M^{-1} \tau_{1}=\tau_{1}, \quad M^{-1} \tau_{2}=\tau_{2}
$$

If (5) holds, then $r=r_{0}=2 \alpha$, and $M^{2}=I$, since $M^{2} \tau_{1}=\tau_{1}$. If (6) holds, then $\tau_{2}=\bar{\tau}_{1} \neq \tau_{1}$ and $M$ has the form (1). Since $\left(a-c \tau_{1}\right)^{\alpha}\left(a-c \tau_{2}\right)^{r-\alpha}=1$ we can conclude as before that $\kappa^{r / 2-\alpha}=1$. Thus $M^{r / 2-\alpha}=I$, if $r$ is even; $M^{r-2 \alpha}=I$ if $r$ is odd.

The statement of the theorem now follows.

THEOREM 8. Let $p(\tau)=A\left(\tau-\tau_{0}\right)^{r_{0}}, r_{0} \leqq r$.

(a) If $r=2 r_{0}$ and $\tau_{0}$ is real, then $\Gamma$ contains at most hyperbolic elements fixing $\tau_{0}$ and $\infty$, and elliptic elements of order two interchanging $\tau_{0}$ and $\infty$.

(b) If $r=r_{0}$ and $\tau_{0}$ is real, then $\Gamma$ consists of at most parabolic elements fixing $\tau_{0}$.

(c) If $r=r_{0}$ and $\tau_{0}$ is not real, $\Gamma$ contains at most elliptic elements of order $r\left(r / 2\right.$, if $r$ is even) fixing $\tau_{0}$.

(d) If $r_{0}=0$, then $\Gamma$ contains at most parabolic elements fixing $\infty$ (translations).

(e) Otherwise $\Gamma=\{I\}$.

In case (c), $\Gamma$ is finite and therefore discontinuous. In cases (b) and (d), if $\Gamma$ is discontinuous it must be cyclic.

Proof. Without loss of generality assume that $A=1$. Let

$$
M=\left(\begin{array}{ll}
a & b \\
c & d
\end{array}\right) \in \Gamma \text {. }
$$

Then the transformation formula gives

or

$$
(c \tau+d) r\left(\frac{a \tau+b}{c \tau+d}-\tau_{0}\right)^{r_{0}}=\left(\tau-\tau_{0}\right)^{r_{0}},
$$

$$
(c \tau+d)^{r-r_{0}}\left\{\tau\left(a-c \tau_{0}\right)+b-d \tau_{0}\right\}^{r_{0}}=\left(\tau-\tau_{0}\right)^{r_{0}} .
$$

Case (d) is trivial. Therefore we may assume $r_{0}>0$. Suppose first that $r \neq r_{0}$. Then we have either

$$
a-c \tau_{0}=0, r=2 r_{0}, c^{r-r_{0}}\left(b-d \tau_{0}\right)^{r_{0}}=1, \tau_{0}=-d / c
$$

or

$$
c=0, d^{r-r_{0}} a^{r_{0}}=1, M^{-1} \tau_{0}=\tau_{0}
$$


If (7) holds, then $(b c-a d)^{r_{0}}=c^{r-r_{0}}\left(b-d \tau_{0}\right)^{r_{0}}=1$. But $b c-a d=-1$, so that $r_{0}$ must be even. Also

$$
M=\left(\begin{array}{rr}
-d & b \\
c & d
\end{array}\right)
$$

$M^{2}=I, M \tau_{0}=\infty$. If (8) holds, then $a=1 / d$, so that $d^{r-2 r_{0}}=1$. If $r-2 r_{0} \neq 0$, then $d=a= \pm 1$, and $M$ is a translation. This contradicts $M^{-1} \tau_{0}=\tau_{0}$. Hence $r=2 r_{0}$, and

$$
M=\left(\begin{array}{ll}
1 / d & b \\
0 & d
\end{array}\right)
$$

is a hyperbolic transformation fixing $\tau_{0}$ and $\infty$.

Suppose now that $r=r_{0}$. Then $a-c \tau_{0} \neq 0$ and we get

$$
\left(a-c \tau_{0}\right)^{r}\left(\tau-M^{-1} \tau_{0}\right)^{r}=\left(\tau-\tau_{0}\right)^{r} .
$$

Hence $M^{-1} \tau_{0}=\tau_{0}$ and $\left(a-c \tau_{0}\right)^{r}=1$. Suppose $\tau_{0}$ is real; then $M$ is either parabolic or hyperbolic. If $M$ is hyperbolic, let $\tau_{1}$ be the other fixed point of $M$. Then $M$ has the form

$$
M=\left(\begin{array}{ll}
a & d \\
c & d
\end{array}\right)=\left[\begin{array}{cc}
\frac{\tau_{0}-\kappa \tau_{1}}{\kappa^{1 / 2}\left(\tau_{0}-\tau_{1}\right)} & \frac{\tau_{0} \tau_{1}(\kappa-1)}{\kappa^{1 / 2}\left(\tau_{0}-\tau_{1}\right)} \\
\frac{(1-\kappa)}{\kappa^{1 / 2}\left(\tau_{0}-\tau_{1}\right)} & \frac{\kappa \tau_{0}-\tau_{1}}{\kappa^{1 / 2}\left(\tau_{0}-\tau_{1}\right)}
\end{array}\right]
$$

The condition $\left(a-c \tau_{0}\right)^{r}=1$ becomes $\kappa^{r / 2}=1$. Hence $M^{r}=I$, contradicting the fact that $M$ is hyperbolic. Hence $M$ must be a parabolic transformation leaving $\tau_{0}$ fixed.

If $\tau_{0}$ is not real, then $M$ must be elliptic. By the above calculation, $M^{r / 2}=I$, if $r$ is even and $M^{r}=I$ if $r$ is odd.

In case (c) $\Gamma$ is finite by Burnside's Theorem (cf. Corollary 6 ). In case (b), $\Gamma$ is isomorphic with an additive subgroup of the real numbers, by the mapping

$$
M \rightarrow \beta, \text { where } M \tau=\tau^{\prime}, \frac{1}{\tau^{\prime}-\tau_{0}}=\frac{1}{\tau-\tau_{0}}+\beta .
$$

The additive subgroups of the real line are either everywhere dense or cyclic. Thus if $\Gamma$ is discontinuous it must be cyclic.

REMARK. It is straightforward exercise to show that polynomial automorphic forms on groups $\Gamma$ of the types described in Theorems 7 and 8 actually do exist. Thus there are polynomial automorphic forms on both discontinuous and nondiscontinuous groups.

5. Multiplier system $v \not \equiv 1$. We are now in a position to discuss automorphic forms with multiplier systems $v \neq \equiv 1$ on nondiscontinuous groups $\Gamma$. As before we may restrict our attention to the case $r \geqq 0$. We begin with the simplest case. 
Lemma 9. Suppose $\Gamma$ is not discontinuous and $g(\tau) \not \equiv 0$ is an automorphic form of dimension 0 with multiplier system $v \neq \equiv 1$, on $\Gamma$. Then either

(a) $g(\tau)=e^{c}((\tau-\alpha) /(\tau-\beta))^{\lambda}$, where $c$ and $\lambda \neq 0$ are constants and $\alpha$ and $\beta$ are real, $\alpha \neq \beta$,

(b) $g(\tau)=e^{c}((\tau-\alpha) /(\tau-\bar{\alpha}))^{\lambda}$, where $c$ is an arbitrary constant, $\lambda \neq 0$ is an integer, and $\bar{\alpha} \neq \alpha$,

(c) $g(\tau)=e^{c} \cdot \exp [-1 / \gamma(\tau-\alpha)]$, where $\alpha$ is real and $c$ and $\gamma \neq 0$ are constants, or

(d) $g(\tau)=e^{c} \cdot e^{\tau / \gamma}$, where $c$ and $\gamma \neq 0$ are constants.

In case (a), $\Gamma$ consists solely of hyperbolic transformations leaving $\alpha$ and $\beta$ fixed. In case (b) $\Gamma$ consists solely of elliptic transformations fixing $\alpha$ and $\bar{\alpha}$. In case (c), $\Gamma$ consists entirely of parabolic transformations leaving $\alpha$ fixed. In case (d), $\Gamma$ consists entirely of parabolic transformations leaving $\infty$ fixed.

Proof. Since $g(\tau)$ is an automorphic form of dimension 0, with multiplier system $v$, by Corollary $3 g(\tau) / g^{\prime}(\tau)$ is an automorphic form of dimension 2, with multiplier system $\equiv 1$. Since $\Gamma$ is not discontinuous it follows from Theorem 1 and Lemma 4 , that $g(\tau) / g^{\prime}(\tau)=p(\tau)$, a polynomial of degree at most two.

Suppose $p(\tau)=\gamma(\tau-\alpha)(\tau-\beta), \alpha \neq \beta$. Then a simple integration yields

$$
g(\tau)=e^{c}\left(\frac{\tau-\alpha}{\tau-\beta}\right)^{\lambda}, \lambda=\{\gamma(\alpha-\beta)\}^{-1} .
$$

Suppose $M \in \Gamma$; then, by considering the zero and the singularlity of $g(\tau)$, we conclude that $M \alpha=\alpha, M \beta=\beta$. Hence $\alpha$ and $\beta$ are real and $M$ is hyperbolic, or $\beta=\bar{\alpha}$ and $M$ is elliptic. In the latter case if $\lambda$ is not an integer, then $g(\tau)$ is not single-valued and hence not meromorphic in $\mathscr{H}$. This is contrary to assumption and cases (a) and (b) are completed.

Suppose $p(\tau)=\gamma(\tau-\alpha)$. Then we get $g(\tau)=e^{c}(\tau-\alpha)^{1 / \gamma}$. A simple calculation shows that if $M \neq I, g(\tau)$ does not have the transformation property of an automorphic form of dimension 0 with respect to $M$. That is, if $(M \tau-\alpha)^{1 / \gamma}=v(M)(\tau-\alpha)^{1 / \gamma}$, then $M=I$. Therefore this case is excluded.

The next possibility is $p(\tau)=\gamma(\tau-\alpha)^{2}$. Then $g(\tau)=e^{c} \cdot \exp [-1 / \gamma(\tau-\alpha)]$. Since $p(\tau)$ is a form of dimension 2 and $\Gamma$ is not discontinuous, it follows from Theorem 8 that $\alpha$ is real and $\Gamma$ consists of parabolic elements leaving $\alpha$ fixed.

Finally, if $p(\tau)=\gamma$, then $g(\tau)=e^{r} e^{\tau / \gamma}$. By Theorem $8, \Gamma$ contains at most translations.

REMARK. This lemma gives necessary conditions for the existence of automorphic forms of dimension 0 on nondiscontinuous $\Gamma$. While these conditions are not quite sufficient, we obtain sufficient conditions (which are incidentally also necessary) by making minor additions. For example, in case (c) the conditions given are sufficient to ensure that $g(M \tau)=v(M) g(\tau)$, for all parabolic $M$ fixing $\alpha$. However $|v(M)| \equiv 1$ if and only if $\gamma=i y$, with $y$ real. 
THEOREM 10. Suppose $\Gamma$ is not discontinuous and $f(\tau) \not \equiv 0$ is an automorphic form of integral dimension $r>0$, with multiplier system $v$, not necessarily $\equiv 1$, on $\Gamma$. Then

$$
f(\tau)^{2 r+2}=p(\tau)^{r} \cdot g(\tau)
$$

where $g(\tau)$ is either a constant or one of the four types (a), (b), (c), (d) of Lemma 9, and $p(\tau)$ is a polynomial automorphic form of dimension $2 r+2$ (and hence of degree at most $2 r+2)$, with at most two distinct zeros.

If $p(\tau)$ has one zero $\tau_{0}$, then $\tau_{0}$ is real and $g(\tau)$ is constant or has the form (c). If $g(\tau)$ is constant then $\Gamma$ is one of two types:

(i) hyperbolic elements fixing $\tau_{0}$ and $\infty$, and elliptic elements of order two interchanging $\tau_{0}$ and $\infty$,

(ii) parabolic elements leaving $\tau_{0}$ fixed.

If $g(\tau)$ has the form (c), $\Gamma$ is of type (ii).

If $p(\tau)$ has two zeros $\tau_{1}$ and $\tau_{2}$, then either $\tau_{1}$ and $\tau_{2}$ are real or $\tau_{2}=\bar{\tau}_{1} \neq \tau_{1}$. If $\tau_{1}$ and $\tau_{2}$ are real, then $g(\tau)$ is constant or has the form (a). If $g(\tau)$ is constant then $\Gamma$ is of the form:

(iii) hyperbolic elements fixing $\tau_{1}$ and $\tau_{2}$ and elliptic elements of order two interchanging $\tau_{1}$ and $\tau_{2}$.

If $g(\tau)$ has the form (a), $\Gamma$ is of type (iii) but cannot contain any elliptic elements. If $\tau_{2}=\bar{\tau}_{1} \neq \tau_{1}$, then $g(\tau)$ is constant or has the form (b). In either case $\Gamma$ has the form

(iv) elliptic elements fixing $\tau_{1}$ and $\tau_{2}$.

If $p(\tau)$ has no zeros, then $g(\tau)$ is constant or has the form (d). In either case $\Gamma$ has the form:

(v) parabolic elements fixing $\infty$.

Whenever $g(\tau)$ is a constant, $v^{2 r+2} \equiv 1$ on $\Gamma$, and conversely.

Proof. Suppose $f(\tau)$ is an automorphic forrm of integral dimension $r>0$, with multiplier system $v$ on $\Gamma$, where $\Gamma$ is not discintinuous. Then $g(\tau)=f(\tau)^{r+2}\left\{f^{(r+1)}(\tau)\right\}^{r}$ is an automorphic form of dimension 0 , with multiplier sysyem $v^{2 r+2}$ on $\Gamma$. On the other hand, $f(\tau) \mid f^{(r+1)}(\tau)$ is an automorphic form of dimension $2 r+2$, with multiplier system $\equiv 1$. Hence by Theorem 1 and Lemma $4, f(\tau) / f^{(r+1)}(\tau)=p(\tau)$, a polynomial of degree at most $2 r+2$. We conclude that $f(\tau)^{2 r+2}=p(\tau)^{r} \cdot g(\tau)$.

Since $\Gamma$ is not discontinuous, it follows from Theorem $A$ that if $v^{2 r+2} \equiv 1$, $g(\tau)$ is constant. On the other hand if $v^{2 r+2} \not \equiv 1, g(\tau)$ is a function of one of the four types (a), (d), (c), (d) of Lemma 9. By Corollary 6, $p(\tau)$ has at most two zeros.

The theorem now follows by considering separately the cases when $p(\tau)$ has no zero, one zero or two zeros, and by applying Theorems 7 and 8 and Lemma 9.

Remarks. 1. If we assume tae dimension of $f(\tau)$ is an integer $r<0$, then we conclude that $f(\tau)^{2 r+2}=\left\{p(\tau)^{r} \cdot g(\tau)\right\}^{-1}$, with the same possibilities for $p(\tau)$ and 
$g(\tau)$ as given in the theorem. The groups $\Gamma$ which can occur are of course the same ones as in the theorem.

2. Again it is a simple matter to verify that forms on nondiscontinuous groups of the type described in Theorem 10 actually do exist.

6. Arbitrary real dimension. Unless otherwise specified, throughout this final section $\Gamma$ is a nondiscontinuous group of real linear fraction transformations, $r$ is an arbitrary real number, and $f(\tau)$ is a nonconstant automorphic form of dimension $r$ on $\Gamma$ (with multiplier system $v$ ).

LeMma 11. $f(\tau)$ has at most one zero in $\mathscr{H}$.

Proof. Suppose $f\left(\tau_{1}\right)=0$, with $\tau_{1} \in \mathscr{H}$. Then, for

$$
V=\left(\begin{array}{ll}
* & * \\
c & d
\end{array}\right) \in \Gamma,
$$

we have $f\left(V \tau_{1}\right)=v(V)\left(c \tau_{1}+d\right)^{-r} f\left(\tau_{1}\right)=0$. Since $\Gamma$ is not discontinuous there is a sequence of distinct $V_{n} \in \Gamma$ such that $V_{n} \rightarrow I$. Now $V_{n} \tau_{1} \rightarrow \tau_{1}$ and at the same time, $f\left(V_{n} \tau_{1}\right)=0$. Since $f$ is meromorphic at $\tau_{1}$, we conclude that $V_{n} \tau_{1}$ takes only finitely many distinct values. With a finite number of exceptions, therefore, $V_{n} \tau_{1}=\tau_{1}$. Thus for almost all $n, V_{n}$ is an elliptic transformation fixing $\tau_{1}$.

Suppose $\tau_{2} \in \mathscr{H}$ and $f\left(\tau_{2}\right)=0$. The above argument shows that for almost all $n, V_{n} \tau_{2}=\tau_{2}$. Therefore $\tau_{2}=\tau_{1}$, and $f(\tau)$ has at most one zero in $\mathscr{H}$.

Corollary 12. $f(\tau)$ has at most one pole in $\mathscr{H}$. If $f(\tau)$ has a zero in $\mathscr{H}$, it has no pole in $\mathscr{H}$.

Proof. Consider $g(\tau)=1 / f(\tau)$, an automorphic form on $\Gamma$ of dimension $-r$, with multiplier system $\bar{v}=1 / v$. By Lemma $11, g(\tau)$ has at most one zero in $\mathscr{H}$; thus $f(\tau)$ has at most one pole in $\mathscr{H}$.

Suppose $\tau_{1}$ is a zero of $f$ and $\tau_{2}$ is a pole of $f$, with $\tau_{1}, \tau_{2} \in \mathscr{H}$. The argument of Lemma 11 shows that for a sequence of distinct $V_{n} \in \Gamma$ such that $V_{n} \rightarrow I$, $V_{n} \tau_{1}=\tau_{1}$ for almost all $n$. The same argument applied to $1 / f(\tau)$ shows that $V_{n} \tau_{2}=\tau_{2}$ for almost all $n$. Hence $\tau_{1}=\tau_{2}$, a contradicion.

THEOREM 13. If $f(\tau)$ has a zero or pole in $\mathscr{H}$, say $\tau_{0}$, then $\Gamma$ consists exclusively of elliptic elements fixing $\tau_{0}$.

If $f(\tau)$ has no zero or pole in $\mathscr{H}$, then $\Gamma$ has one of the following forms:

(i) hyperbolic elements all fixing two given distinct points $\tau_{1}$ and $\tau_{2}$, and elliptic elements of order two interchanging $\tau_{1}$ and $\tau_{2}\left(\tau_{2}=\infty\right.$ is not excluded).

(ii) parabolic elements all fixing a given real point $\tau_{1}\left(\tau_{1}=\infty\right.$ is not excluded).

Proof. Suppose $\tau_{0} \in \mathscr{H}$ and $f\left(\tau_{0}\right)=0$. Then by Lemma $11 f$ has no other zero in $\mathscr{H}$. Therefore for $V \in \Gamma, V \tau_{0}=\tau_{0}$, since $f\left(V \tau_{0}\right)=0$. If $\tau_{0} \in \mathscr{H}$ is a pole of $f$, the same argument applied to $1 / f(\tau)$ shows that $V \tau_{0}=\tau_{0}$, for all $V \in \Gamma$. 
If $f(\tau)$ has no zero and no pole in $\mathscr{H}$, then $\log f(\tau)$ is well defined in $\mathscr{H}$, after a branch is chosen. Thus any power of $f$ is meromorphic in $\mathscr{H}$. But then $f^{1 / r}$ is an automorphic form on $\Gamma$ of dimension 1 . The rest follows from an application of Theorem 10.

We now drop the assumption that $\Gamma$ is nondiscontinuous and obtain the following result as an immediate consequence if Theorem 13.

Corollary 14. Suppose $\Gamma$ preserves $\mathscr{H}$ and $\Gamma$ has translations (i.e. parabolic elements leaving $\infty$ fixed) and at least one nontranslation. If there exists a nonconstant automorphic form of real dimension on $\Gamma$, then $\Gamma$ is discontinuous.

\section{BIBLIOGRAPHY}

1. G. Bol, Invarianten linearer Differentialgleichungen, Abh. Math. Sem. Univ. Hamburg 16 (1949), 1-28.

2. C. W. Curtis and I. Reiner, Representation theory of finite groups and associative algebras, Interscience, New York, 1962.

3. J. Lehner, Discontinuous groups and automorphic functions, Math. Surveys No. 8, Amer. Math. Soc., Providence, R. I., 1964.

THE UNIVERSITY OF WISCONSIN

Madison, Wisconsin 